\title{
Magnitude and correlates of virological failure among adult HIV patients receiving PI based second line ART regimens in north western Tanzania; a case control study
}

Daniel W. Gunda ${ }^{1,2^{*}}$ (D), Semvua B. Kilonzo ${ }^{1,2}$, Tarcisius Mtaki ${ }^{2}$, Desderius M. Bernard ${ }^{2}$, Samwel E. Kalluvya ${ }^{1,2}$ and Elichilia R. Shao ${ }^{3}$

\begin{abstract}
Background: With a growing access to free ART, switching of ART to second line regimen has also become common following failure of first line ART regimens. Patients failing on first line ART regimens have been shown to stand a high risk of failing on subsequent second line ART regimens. The magnitude of those who are failing virologicaly on second line ART is not documented in our setting. This study was designed to assess the magnitude and correlates of second line ART treatment failure.

Methods: A retrospective analysis of patients on second line ART for at least 1 year was done at Bugando care and treatment center. Information on demographic, clinical and laboratory data were collected and analyzed using STATA 11. The proportion of patients with Virological failure was calculated and potential correlates of virological failure were determined by logistic regression model.

Results: In total 197 patients on second line ART were included in this study and 24 (12.18\%) of them met criteria for virological failure. The odds of having virological failure on second line ART were independently associated with age of less than 30 years ( $A O R=12.5, p=0.001$ ), being on first line for less than 3 years $(A O R=6.1, p=0.002)$ and $C D 4$ at switch to second line ART of less than 200cells/ $\mu \mathrm{l}(\mathrm{AOR}=16.3, p<0.001)$.

Conclusion: Virological failure among patients on second line ART is common. Predictors of virological failure in this study could assist in planning for strategies to improve the outcome of this subgroup of patients including close clinical follow up of patients at risk, a continued adherence intensification and a targeted resistance testing before switching to second line ART.
\end{abstract}

Keywords: Second line ART, Virological failure on second line ART, Northwestern Tanzania

\section{Background}

The patients experiencing first-line antiretroviral therapy (ART) treatment failure have been shown to stand a higher risk of failing on subsequent second line regimens due to side effects and complex regimens which could lead into selection of resistant strains [1]. These patients could potentially transmit the resistant strains which are a big

\footnotetext{
* Correspondence: daniel_rev2002@yahoo.com

'Department of Medicine, Bugando Medical centre, 1370 Mwanza, Tanzania ${ }^{2}$ Department of medicine, Weill Bugando School of Medicine, 1464 Mwanza, Tanzania

Full list of author information is available at the end of the article
}

challenge in resource limited settings like ours where resistance testing is not practicable [2, 3]. A better strategy for HIV control would in this manner necessitate a durable and potent ART regimen. Furthermore this also requires early detection and appropriate revision of the failing ART regimens and timely switch to potent second line ART regimens $[4,5]$.

Switching to potent second line ART regimens has been shown to have a number of benefits including improving virological containment and immune response [6-8], reduced probable drug resistance [9] and reduced mortality [10]. Even with these advantages still a proportion of

(c) The Author(s). 2019 Open Access This article is distributed under the terms of the Creative Commons Attribution 4.0 International License (http://creativecommons.org/licenses/by/4.0/), which permits unrestricted use, distribution, and reproduction in any medium, provided you give appropriate credit to the original author(s) and the source, provide a link to the Creative Commons license, and indicate if changes were made. The Creative Commons Public Domain Dedication waiver (http://creativecommons.org/publicdomain/zero/1.0/) applies to the data made available in this article, unless otherwise stated. 
patients have been reported elsewhere to experience treatment failure while on second line ART regimen with varying magnitudes of this problem [11-13]. Virological failure on first line ART has been associated with poor adherence and its persistent may seriously limit the durability of second line ART regimen [14]. Second line ART regiments are expensive and usually more complex and are potentially associated with higher levels of side effects which may complicate non adherence and increase the risk of resistance and virological failure [15]. Other reported predictors of virological failure include WHO clinical stage $3 \& 4$ and severe immune suppression at switch to second line ART [16], and falling CD4 counts on receipt of second line ART has been shown to have a strong reciprocal relation to virological improvement [17] among others.

The literature on the extent of this problem in our setting is still scarce. This study therefore was designed to determine the prevalence and assess the risk factors of treatment failure among adult HIV patients who are receiving protease inhibitor (PI) based second line ART regimen in northwestern Tanzania. This information is important in devising strategies to optimize the outcome of this subgroup of patients but we also believe it will significantly add to the existing body of knowledge on virological performance of PI based second line ART regimen in resource limited settings like ours.

\section{Methods}

This was a case control study which was done during elective research period from September 2017 to May 2018 at Bugando medical center (BMC) HIV Care and treatment center (CTC). BMC is a tertiary level and a university teaching hospital located in the North Western part of Tanzania. Currently the hospital has a capacity of 1000 beds, and serving about 16 million people. As one of its core services BMC also runs HIV activities with a referral CTC department. The center started a way back 2004, and it currently serves a total of more than 14,000 clients. Core activities at CTC include provision of ART, follow up and treatment monitoring. ART drugs are provided for free of charge and about 10,000 patients are currently on ART. In our setting first line ART are basically Non Nucleoside/ Nucleotide Reverse Transcriptase Inhibitors (NNRTI) based regimens; whereas patients failing on first line ART regimens are usually switched to Protease Inhibitor (PI) based second line ART regimen. Around 500 patients are currently receiving PI based second line ART regimen. ART treatment monitoring is routinely done using clinical and immunological parameters where CD4 counts are checked 6 monthly since viral loads (VL) are still expensive for routine use. Targeted VL and adherence intensification are usually done before switching to second line ART is done. Patient with VL of more than 1000copies/ml usually get a repeat testing after 3 months of adherence intensification and those with log drop of less than 0.5 are classified as failing virologicaly. Our current cohort of patients on PI based second line ART had a regular VL test 6 monthly. Those found with VL of more than 1000copies/ $\mu$ l had adherence intensification and a conclusion of virological failure was reached if a subsequent VL was more than 1000copies/ $\mu$ l. The adherence was assessed by a combination of patients' report and pill count as done previously [18] where adherence rate of less than 95\% denotes poor adherence. In our setting as it is also true for most of the resource limited settings no resistance testing is done prior switching to second line ART regimen to decrease possibilities of cross resistance.

The study involved all adult HIV positive patients who are currently receiving PI based second line ART regimens at Bugando CTC in Mwanza. A minimum sample size of 178 was estimated from Leslie Kish formula (1965) for cross sectional studies assuming $13.35 \%$ of patients on second line ART will experience virological failure as found previously [19] with an allowable error of 0.05 at $95 \% \mathrm{CI}$. The sample was derived from a list of all patients who were started on second line ART regimen at BMC. A CTC data base was reviewed to identify all patients who were on PI based second line ART and registration numbers were used to trace the files. The files were then numbered to ease sampling. A systematic sampling was done to get the required sample size using a skip interval of 2 where the starting point was randomly selected as done previously [20]. Files of patients who were on PI based second line ART for less than a year and those who were transferred out were not included and the next file was selected in its place. The data regarding age, sex, date of HIV diagnosis, WHO clinical stage, CD4 at initiation of first line ART, year of ART initiation and regimen, VL at switch to second line, time in months on first line, duration in months on second line ART and ART regimen, adherence status, most recent VL, and CD4 counts at switch to second line were extracted.

Data were computerized using Epi data version 3.1 and STATA version 11 (Stata Corp LP, college station, TX) was used for data analysis. Continuous variables were expressed as medians with interquartile range (IQR) while categorical variables were expressed as proportions with percentages. The treatment outcome in this study was defined as development of virological failure as used previously where patients who had a second VL of more than 1000copies/ $\mu$ l while receiving PI based second line ART regimen for at least a year were coded as failing virologicaly [12]. The proportion of patients with virological failure on second line ART was then calculated and expressed as percentage and the effect of different risk factors on the odds of developing virological failure was investigated. The Odds ratio with 95\% Confidence Interval (CI) was calculated using univariate analysis followed by multivariate analysis model to 
assess the association of different variables to the outcome of interest. In all our analysis factors were said to be statistically significant when the $p$ value was less than 0.05 .

\section{Ethical clearance}

The permission to conduct and publish the results from this study was sought from the catholic University of Health and allied Sciences/ Bugando Medical Center joint ethical committee. The patient's files were handled by the researchers alone and the patients' identifiers including names and registration numbers were not included in the analysis to further maintain confidentiality.

\section{Results}

In total 197 patients were enrolled in this study. About 3 quarters, $146(74.11 \%)$ of the study participants were female patients and almost a third of participants, 64 (32.65\%) had lost their spouse. At enrollment to CTC most patients, 114 (57.87\%) had WHO clinical stage 3 \& 4 AIDS defining illnesses and 57 (28.93\%) had severe immune suppression. With a median time on second line ART of 14 [13-18] months, 26 (13.20\%) participants had an associated severe immune suppression at initiation of second line ART and most patients, 135 (68.53\%) were coded as having WHO stage $3 \& 4$. Though most patients had good adherence, still $25(12.69 \%)$ had poor adherence to medication as summarized in Table 1. Overall Zidovudine based regimens were the most common, 105 (53.3\%) first line ART regimen and most patients, 1129 (65.5\%) were subsequently switched to ritonavir boosted Lopinavir based regimen as shown in Figs. 1 and 2 respectively.

A total of $24(12.18 \%)$ participants were found to meet criteria for virological failure in this study (Table 1). The odds of having virological failure on PI based second line ART in this study were independently associated with age of less than 30 years $(\mathrm{AOR}=12.4, p=0.001)$, being on first line ART regimen for less 3 years $(\mathrm{AOR}=7.6, p$ $=0.003$ ) and CD4 counts of less than 200cells $/ \mu$ at switch to second line ART (AOR $=16.3, p<0.001)$. Adherence level of less than $95 \%$ had a significant association with virological failure on univariate analysis alone $(\mathrm{OR}=3.4, p=0.028)$ while the difference in distribution of other variables was not different statistically including the type of ART regimens and time on second line ART as summarized in Table 2.

\section{Discussion}

The objective of this study was to determine the prevalence and risk factors of virological failure among adult HIV patients receiving PI based second ART in northwestern Tanzania. The $12.18 \%$ rate of virological failure on PI based second line ART in this study is similar to prevalence rates of $13.35 \%$ reported earlier in 2012 from South Africa [19] and 13.9\% reported in 2014 by Sigaloff
Table 1 General Characteristics of 197 participants on PI based second line ART regimen

\begin{tabular}{|c|c|c|}
\hline Variable & Frequency & Percent or median (IQR) \\
\hline Age (years) & 197 & $48[41-54]$ \\
\hline \multicolumn{3}{|l|}{ Sex } \\
\hline Male & 51 & 25.89 \\
\hline Female & 146 & 74.11 \\
\hline \multicolumn{3}{|l|}{ Marital status } \\
\hline Married & 56 & 28.43 \\
\hline Divorce & 36 & 18.27 \\
\hline Widow & 64 & 32.65 \\
\hline single & 40 & 20.30 \\
\hline \multicolumn{3}{|l|}{ WHO stage at ART1 } \\
\hline $3 \& 4$ & 114 & 57.87 \\
\hline $1 \& 2$ & 83 & 42.13 \\
\hline \multicolumn{3}{|l|}{ CD4 at ART1 } \\
\hline Median (cells/ul) & 197 & 295.5 [179-406] \\
\hline 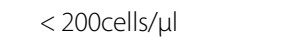 & 57 & 28.93 \\
\hline$\geq 200$ cells $/ \mu \mathrm{l}$ & 140 & 71.07 \\
\hline \multicolumn{3}{|l|}{ WHO stage at ART2 } \\
\hline $3 \& 4$ & 135 & 68.53 \\
\hline $1 \& 2$ & 62 & 31.47 \\
\hline \multicolumn{3}{|l|}{ CD4 at ART2 } \\
\hline Median (cells/ul) & 197 & 484 [341-700] \\
\hline$<200 \mathrm{cells} / \mu \mathrm{l}$ & 26 & 13.20 \\
\hline$\geq 200 \mathrm{cells} / \mu \mathrm{l}$ & 171 & 86.80 \\
\hline First line ART months & 197 & 113 [46-133] \\
\hline Second line ART months & 197 & 14 [13-18] \\
\hline \multicolumn{3}{|l|}{ Adherence $<95 \%$} \\
\hline Yes & 25 & 12.69 \\
\hline No & 172 & 87.31 \\
\hline \multicolumn{3}{|l|}{ 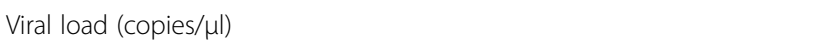 } \\
\hline$\geq 1000$ & 24 & 12.18 \\
\hline$<1000$ & 173 & 87.82 \\
\hline
\end{tabular}

CD4 Cluster of differentiation 4, CD4 at ART1 denotes CD4s at initiation of first line ART, CD4 at ART2 CD4 counts at switch to second line ART, IQR Interquatile range, WHOs World health Organization clinical stage, WHO stage at ART1 denotes WHO clinical stage at initiation of first line ART, WHO stage at ART2 Denotes WHO clinical stage at switch to second line ART

among sub-Saharan patients [21]. Another comparatively higher rate of virological failure of $17.7 \%$ was reported in India among patients who were on second line ART beyond 1 year [12] and in South Africa where it was shown that about $23 \%$ of patients who failed on first line ART experienced virological failure after 1 year of treatment with second line ART [11]. A much higher rate of virological failure of $37 \%$ was also recently reported from Myanmar Asia among patient who were on second line ART [22]. 


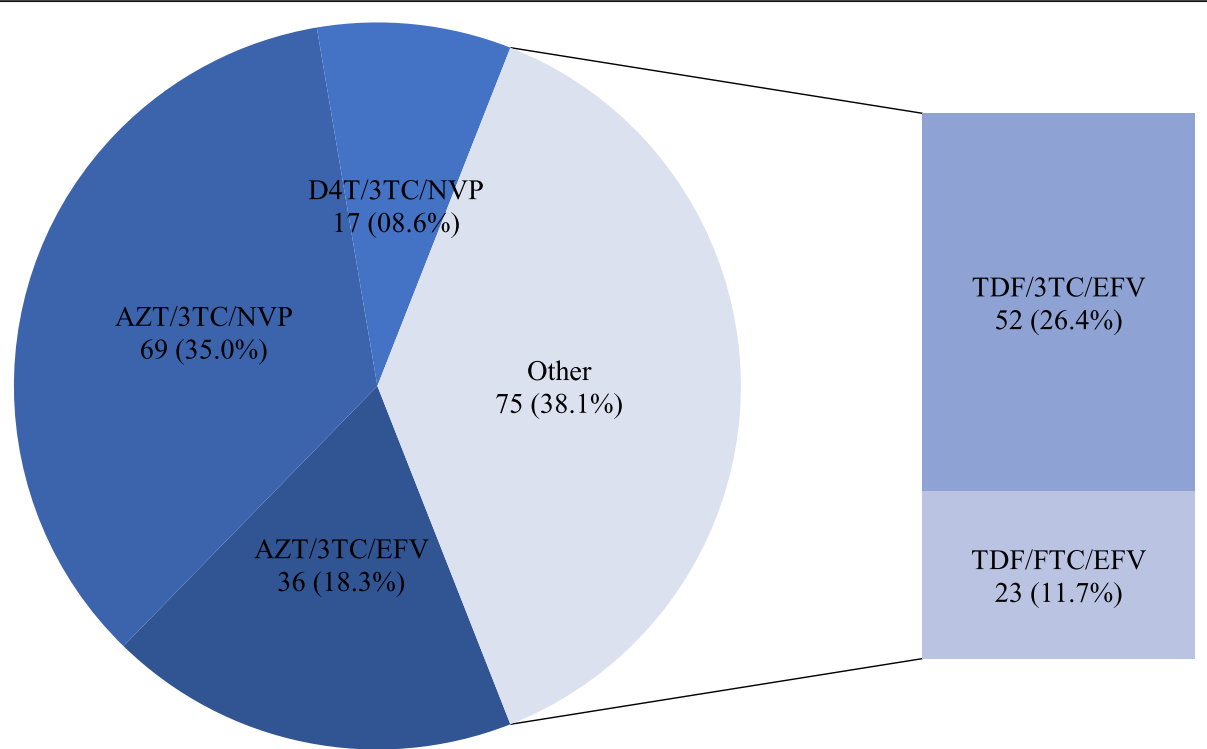

Fig. 1 Distribution of first line ART regimen among 197 adult HIV positive participants. AZT: Zidovudine, 3TC: Lamivudine, D4T: Stavudine, EFV: Efavirenz, FTC, Emtricitabine, NVP: Nevirapine, TDF: Tenofovir

Though the rate of virological failure in the index study is comparatively lower than findings from elsewhere, in general all these findings suggest that virological failure is a common phenomenon with a potential threat to second line long term outcome. One systematic review in resource limited setting indicated that virological failure is a common encounter among HIV patients on second line ARTs which tend to increase in magnitude with time on ART suggesting a possible accumulation of resistant strains as a cause of this treatment failure [23].

Several factors were investigated for possible independent association with virological failure in the index study.
In our study we found that patients who experienced treatment failure after being on first line ART for less than 36 months were more likely to fail on subsequent PI based second line ART. A similar observation was reported by Boerma in 2017 indicating that of those patients who failed on first line ART regimen less than 24 months were also more likely to fail on second line ART [24]. This could be because of possible cross resistance mutations in this subgroup of patients as suggested previously $[25,26]$. In Mozambique it was shown that resistance mutations were a common cause of virological failure on first line ARTs, the prevalence of which kept on increasing with

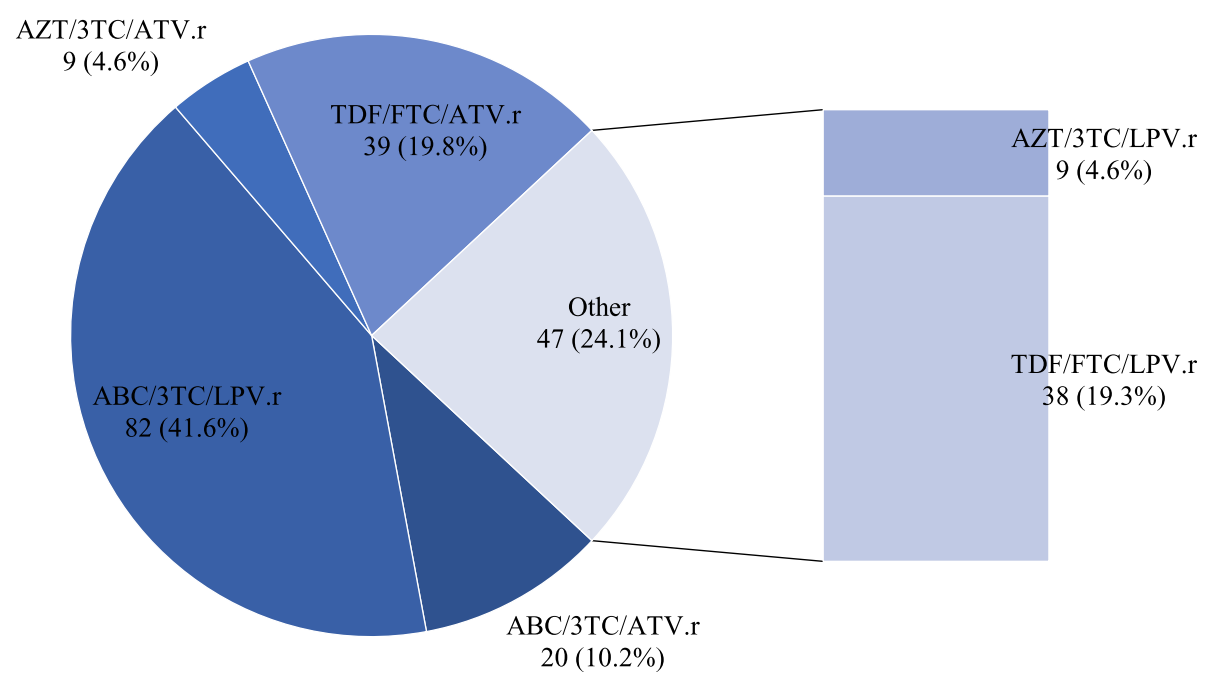

Fig. 2 Distribution of PI based second line ART regimen among 197 adult HIV positive participants. ABC: Abacavir, AZT: Zidovudine, ATV.r: ritonavir boosted Atazanavir, 3TC: Lamivudine, FTC, Emtricitabine, LPV.r: ritonavir boosted Lopinavir, TDF: Tenofovir 
Table 2 Univariate and multivariate analysis for factors associated with virological failure among 197 adult HIV patients on PI based second line ART

\begin{tabular}{|c|c|c|c|c|c|c|}
\hline \multirow[t]{2}{*}{ Variables } & \multicolumn{2}{|c|}{ Virological failure } & \multicolumn{2}{|l|}{ Unadjusted } & \multicolumn{2}{|l|}{ Adjusted } \\
\hline & No $(n \%)$ & Yes $(n \%)$ & OR $(95 \% \mathrm{Cl})$ & $P$-value & OR $(95 \% \mathrm{Cl})$ & $P$-value \\
\hline \multicolumn{7}{|l|}{ Sex } \\
\hline Male & $042(24.28)$ & $09(37.50)$ & & & & \\
\hline Female & $131(75.72)$ & $15(62.50)$ & $1.9(0.7-4.5)$ & 0.171 & & \\
\hline \multicolumn{7}{|l|}{ Age } \\
\hline$<30$ years & $012(06.94)$ & $07(29.17)$ & & & & \\
\hline$\geq 30$ years & 161 (93.06) & $17(70.83)$ & $5.5(1.9-15.9)$ & 0.002 & $12.4(2.7-56.1)$ & 0.001 \\
\hline \multicolumn{7}{|l|}{ Marital state } \\
\hline Married & $46(26.59)$ & $10(41.67)$ & $1.9(0.8-4.7)$ & 0.130 & - & - \\
\hline Divorced & $33(19.08)$ & $03(12.50)$ & $0.6(0.2-2.2)$ & 0.439 & - & - \\
\hline Widow & $59(34.30)$ & 05 (20.83) & $0.5(0.3-1.4)$ & 0.194 & - & - \\
\hline Single & $34(19.65)$ & $06(25.00)$ & $1.3(0.5-3.6)$ & 0.543 & - & - \\
\hline \multicolumn{7}{|c|}{ WHO stage at ART1 } \\
\hline $3 \& 4$ & $101(58.38)$ & $13(54.17)$ & & & & \\
\hline $1 \& 2$ & $072(41.62)$ & $11(45.83)$ & $0.8(0.3-2.0)$ & 0.695 & - & - \\
\hline \multicolumn{7}{|l|}{ ART1months } \\
\hline$\leq 36$ months & 015 (08.67) & 08 (33.33) & & & & \\
\hline$>36$ months & $158(91.33)$ & $16(66.67)$ & $5.3(1.9-14.3)$ & 0.001 & $7.6(1.9-29.0)$ & 0.003 \\
\hline \multicolumn{7}{|l|}{ CD4 at ART2 } \\
\hline 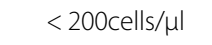 & $035(20.23)$ & $18(75.00)$ & & & & \\
\hline$\geq 200$ cells $/ \mu \mathrm{l}$ & 138 (79.77) & $06(25.00)$ & $11.8(4.3-32)$ & $<0.001$ & $16.3(5 .-57.9)$ & $<0.001$ \\
\hline \multicolumn{7}{|c|}{ WHO stage at ART2 } \\
\hline $3 \& 4$ & $121(69.94)$ & $14(58.33)$ & & & & \\
\hline $1 \& 2$ & 052 (30.06) & $10(41.67)$ & $0.6(0.3-1.4)$ & 0.255 & - & - \\
\hline ART2 months & 009 [05-11] & 6 [4.5-9.5] & $0.9(0.7-1.0)$ & 0.065 & - & - \\
\hline \multicolumn{7}{|l|}{ Adherence < 95\% } \\
\hline Yes & 017 (09.83) & 08 (33.33) & & & & \\
\hline No & $156(90.17)$ & $16(66.67)$ & $4.5(2.3-12.3)$ & 0.002 & $3.4(0.9-12.6)$ & 0.062 \\
\hline \multicolumn{7}{|l|}{ ART1rgm } \\
\hline AZT/3TC/EFV & $32(18.50)$ & $04(16.67)$ & $0.9(0.3-2.8)$ & 0.828 & - & - \\
\hline AZT/3TC/NVP & $66(38.15)$ & 03 (12.50) & $0.2(0.1-0.8)$ & 0.022 & - & - \\
\hline D4T/3TC/NVP & $15(08.67)$ & $02(08.33)$ & $0.9(0.2-4.4)$ & 0.956 & - & - \\
\hline TDF/3TC/EFV & $60(34.68)$ & $15(62.50)$ & $3.1(2.1-7.6)$ & 0.011 & $0.5(0.2-1.9)$ & 0.363 \\
\hline \multicolumn{7}{|l|}{ ART2rgm } \\
\hline ABC/3TC/LPV.r & $86(49.71)$ & $16(66.67)$ & $2.1(1.8-4.9)$ & 0.0125 & $2.1(0.5-7.8)$ & 0.272 \\
\hline AZT/3TC/ATV.r & 07 (04.05) & $02(08.33)$ & $2.1(0.4-11)$. & 0.357 & - & - \\
\hline AZT/3TC/LPV.r & $07(04.05)$ & $02(08.33)$ & $2.1(0.4-11)$ & 0.357 & - & - \\
\hline TDF/FTC/ATV.r & 37 (21.39) & $01(04.70)$ & $0.2(0.2-1.2)$ & 0.077 & - & - \\
\hline TDF/FTC/LPV.r & $36(20.81)$ & 03 (12.50) & $0.4(0.2-1.9)$ & 0.346 & - & - \\
\hline
\end{tabular}

ABC Abacavir, ART1rgm first line ART regimen, ART2rgm second line ART regimen, AZT Zidovudine, ATV.r ritonavir boosted Atazanavir, 3TC Lamivudine, CD4 Cluster of differentiation 4, CD4 at ART2 CD4 counts at switch to second line ART, CI Confidence interval, D4T Stavudine, EFV Efavirenz, FTC Emtricitabine, IQR Interquatile range, LPV.r ritonavir boosted Lopinavir, $n$ number, NVP Nevirapine, rgm regimen, TDF Tenofovir, WHO stage at ART1 Denotes World health Organization clinical stage at initiation of first line ART, WHO stage at ART2 Denotes WHO clinical stage at switch to second line ART 
time on ART [27]. This suggests that in settings like ours where switching to second line ART is not routinely guided by resistance testing, a possible selection and accumulation of drug resistant strains could possibly lead into early failure of second line ART regimen [26, 28], though the time on second line ART was not statistically different in our study between those failing virologicaly and their successful counter parts (6 months vs. 9 months, $p=0.065$ ).

Additionally in this study young patients were found to have increased risk of virological failure especially among those who were younger than 30 years. Onoya and colleagues had similar observation indicating that patients who were younger than 40 years were more likely to fail on second line ART [29]. Similar observations were also reported by Boerma et al. in a multicenter analysis of second line ARTs among pediatric patients where adolescents (10-18 years) were found to have an increased risk of failing on second line ART [24]. Though poor adherence to ART medications did not show any independent association with virological failure in this study, previous studies had indicated that poor adherence is associated with virological failure and is more common among younger patients as compared to older patients [30, 31].

Poor adherence would limit virological control and a loss of immunological and clinical improvement [15]. In a recent study by Tsegaye et al. in 2016 it was indicated that patients with virological failure on second line ART regimen independently had severe immune suppression with CD4 counts of less than 100cells/ $\mu \mathrm{l}$ and WHO clinical stage $3 \& 4$ AIDS defining illness at switch to second line ART regimen [16]. This report is in agreement with our finding where we also observed that patients with virological failure were about 16 times more likely to have an associated severe immune suppression at switch to PI based second line ART with CD4 counts of less than 200 cells/ $\mu$ l as compared to their virologicaly successful counterparts though the difference in clinical deterioration was not statistically different between the two groups.

This study is potentially liable to a number of limitations. Being a single center study its results may not be generalizable. It was a retrospective study from which some information could not be retrieved including assessment of attendant opportunistic conditions. However even with these limitations this is probably the first study to evaluate virological performance of PI based second line ART without resistance guidance in Tanzania and thus we think the findings from this study are valuable and will serve as a potential foundation for further studies to maximize the outcome of HIV patients in this part of resource limited setting which is currently serving as CTC referral facility for the northwestern Tanzania. A prospective analysis of patients with probably resistance testing component might give us a better insight of this subgroup of patients.

\section{Conclusion}

In conclusion these findings are clinically important in our setting suggesting that virological failure on present PI base second line ART is a prevailing problem though its size is relatively small in this study as compared to most other studies. Patients failing on second line ART have increased risk of mortality as shown in previous studies [32, 33]. Since third line ART are expensive and are not currently in use in our setting early identification and closer clinical follow up of patients at risk of failing on second line ART would improve their outcome. Patient at risk of virological failure on second line ART include those who failed shortly on first line ART regimen, those who were younger than 30 years. Non improving CD4 counts at switch to second line ART is also a potential marker of a subsequent virological failure in this subgroup of patients. We believe that apart from current continued adherence intensification, a targeted resistance testing might serve as a potential strategy in further improving the outcome of this subgroup of patients in our setting where third line options are still unavailable.

\begin{abstract}
Abbreviations
3TC: Lamivudine; ABC: Abacavir; AIDS: acquired immune deficiency syndrome; ART: antiretroviral therapy; ATV.r: ritonavir boosted Atazanavir; AZT: Zidovudine; BMC: Bugando medical center; CD4: cluster of differentiation 4; Cl: confidence interval; CTC: care and treatment Center; CUHAS: Catholic University of Health and Allied Sciences; D4T: Stavudine; EFV: Efavirenz; FTC: Emtricitabine; IQR: Interquatile range; LPV.r: ritonavir boosted Lopinavir; NVP: Nevirapine; Ols: opportunistic infections; OR: odds ratio; PI: protease inhibitor; TDF: Tenofovir; VL: viral load; WHO: World Health Organization
\end{abstract}

\section{Acknowledgments}

The authors would like to acknowledge the support given by all staff members at Bugando HIV care and treatment center.

Consent to for publication

Not applicable.

Funding

No funding was received for this study.

Availability of data and materials

We declare that the supporting data can be available upon request from the corresponding author.

\section{Authors' contributions}

All authors read and approved the final version of the manuscript. DWG: Designed the study, acquired and analyzed the data, interpreted the data and did manuscript drafting, SBK: assisted designing the study, assisted with data interpretation, and critically reviewed the manuscript for its intellectual content; TM: assisted with data collection, and critically reviewed the manuscript for its intellectual content, BMD: assisted acquiring the data, assisted with data interpretation, and critically reviewed the manuscript for its intellectual content, SEK: assisted designing the study, assisted with data interpretation, and critically reviewed the manuscript for its intellectual content; ERS: assisted data analysis and critically reviewed the manuscript for its intellectual content.

\section{Ethics approval and consent to participate}

The permission to conduct and publish the results from this study was sought from the catholic University of Health and allied Sciences/ Bugando Medical Center joint ethical committee with clearance certificate no 515/ 2017. The patient's files were handled by the researchers alone and the 
patients' identifiers including names and registration numbers were not included in the analysis to further maintain confidentiality.

\section{Competing interests}

The authors declare that they have no competing interests.

\section{Publisher's Note}

Springer Nature remains neutral with regard to jurisdictional claims in published maps and institutional affiliations.

\section{Author details}

'Department of Medicine, Bugando Medical centre, 1370 Mwanza, Tanzania. 2Department of medicine, Weill Bugando School of Medicine, 1464 Mwanza, Tanzania. ${ }^{3}$ Department of Medicine, Kilimanjaro Christian Medical University College, 2240 Moshi, Tanzania.

\section{Received: 18 August 2018 Accepted: 25 February 2019}

\section{Published online: 07 March 2019}

\section{References}

1. Todd J, et al. Risk factors influencing HIV infection incidence in a rural African population: a nested case-control study. J Infect Dis. 2006;193(3):458-66.

2. Petersen $\mathrm{ML}$, et al. Long-term consequences of the delay between virologic failure of highly active antiretroviral therapy and regimen modification. AIDS. 2008:22(16):2097-106.

3. Kantor R, et al. Misclassification of first-line antiretroviral treatment failure based on immunological monitoring of HIV infection in resource-limited settings. Clin Infect Dis. 2009;49(3):454-62.

4. El-Khatib Z, et al. Adherence to drug-refill is a useful early warning indicator of virologic and immunologic failure among HIV patients on first-line ART in South Africa. PLoS One. 2011;6(3):e17518.

5. Mutevedzi PC, et al. Association of age with mortality and virological and immunological response to antiretroviral therapy in rural south African adults. PLoS One. 2011;6(7):e21795.

6. Ferradini $\mathrm{L}$, et al. High efficacy of lopinavir/r-based second-line antiretroviral treatment after 24 months of follow up at ESTHER/Calmette Hospital in Phnom Penh, Cambodia. J Int AIDS Soc. 2011;14:14.

7. Pujades-Rodriguez $\mathrm{M}$, et al. Immunovirological outcomes and resistance patterns at 4 years of antiretroviral therapy use in HIV-infected patients in Cambodia. Tropical Med Int Health. 2011;16(2):205-13.

8. Patel $\mathrm{D}$, et al. Early outcome of second line antiretroviral therapy in treatment-experienced human immunodeficiency virus positive patients. Perspect Clin Res. 2013;4(4):215-20.

9. Nichols $B E$, et al. Increasing the use of second-line therapy is a cost-effective approach to prevent the spread of drug-resistant HIV: a mathematical modelling study. J Int AIDS Soc. 2014;17:19164.

10. Gsponer T, et al. The causal effect of switching to second-line ART in programmes without access to routine viral load monitoring. AIDS. 2012;26(1):57-65.

11. Fox MP, et al. High rates of survival, immune reconstitution, and virologic suppression on second-line antiretroviral therapy in South Africa. J Acquir Immune Defic Syndr. 2010;53(4):500-6.

12. Chakravarty J, et al. Outcome of patients on second line antiretroviral therapy under programmatic condition in India. BMC Infect Dis. 2015;15:517.

13. van Zyl GU, et al. Low lopinavir plasma or hair concentrations explain second-line protease inhibitor failures in a resource-limited setting. J Acquir Immune Defic Syndr. 2011;56(4):333-9.

14. Ramadhani $\mathrm{HO}$, et al. Association of first-line and second-line antiretroviral therapy adherence. Open Forum Infect Dis. 2014;1(2):ofu079.

15. El-Khatib Z, et al. Viremia and drug resistance among HIV-1 patients on antiretroviral treatment: a cross-sectional study in Soweto, South Africa. AIDS. 2010;24(11):1679-87

16. Tsegaye AT, et al. Predictors of treatment failure on second-line antiretroviral therapy among adults in Northwest Ethiopia: a multicentre retrospective follow-up study. BMJ Open. 2016;6(12):e012537.

17. Ojha CR, Shakya G, Dumre SP. Virological and immunological status of the people living with HIV/AIDS undergoing ART treatment in Nepal. Biomed Res Int. 2016;2016:6817325.

18. Gunda DW, et al. Plasma concentrations of efavirenz and nevirapine among HIV-infected patients with immunological failure attending a tertiary hospital in North-Western Tanzania. PLoS One. 2013;8(9):e75118.
19. Levison $\mathrm{JH}$, et al. Virologic failure of protease inhibitor-based second-line antiretroviral therapy without resistance in a large HIV treatment program in South Africa. PLoS One. 2012;7(3):e32144.

20. Mhozya $\mathrm{H}_{\text {, et }}$ al. Late-stage disease at presentation to an HIV clinic in eastern Tanzania: a retrospective cross-sectional study. Malawi Med J. 2015;27(4):125-7.

21. Sigaloff $\mathrm{KC}$, et al. Second-line antiretroviral treatment successfully resuppresses drug-resistant HIV-1 after first-line failure: prospective cohort in sub-Saharan Africa. J Infect Dis. 2012;205(11):1739-44.

22. Kyaw NTT, et al. Long-term outcomes of second-line antiretroviral treatment in an adult and adolescent cohort in Myanmar. Glob Health Action. 2017; 10(1):1290916.

23. Ajose $\mathrm{O}$, et al. Treatment outcomes of patients on second-line antiretroviral therapy in resource-limited settings: a systematic review and meta-analysis. AIDS. 2012;26(8):929-38.

24. Boerma RS, et al. Multicentre analysis of second-line antiretroviral treatment in HIV-infected children: adolescents at high risk of failure. J Int AIDS Soc. 2017;20(1):21930.

25. Kasang C, et al. HIV drug resistance (HIVDR) in antiretroviral therapy-naive patients in Tanzania not eligible for WHO threshold HIVDR survey is dramatically high. PLoS One. 2011;6(8):e23091.

26. Hawkins C, et al. HIV virological failureand drug resistance in a cohort of Tanzanian HIV-infected adults. J Antimicrob Chemother. 2016;71(7):1966-74.

27. De Luca A, et al. Accumulation of HIV-1 drug resistance in patients on a standard thymidine analogue-based first line antiretroviral therapy after virological failure: implications for the activity of next-line regimens from a longitudinal study in Mozambique. BMC Infect Dis. 2017:17(1):605.

28. Sigaloff KC, et al. Accumulation of HIV drug resistance mutations in patients failing first-line antiretroviral treatment in South Africa. AIDS Res Hum Retrovir. 2012;28(2):171-5.

29. Onoya D, et al. Predicting the need for third-line antiretroviral therapy by identifying patients at high risk for failing second-line antiretroviral therapy in South Africa. AIDS Patient Care STDs. 2017;31(5):205-12.

30. Tsega B, Srikanth BA, Shewamene Z. Determinants of non-adherence to antiretroviral therapy in adult hospitalized patients, Northwest Ethiopia. Patient Prefer Adherence. 2015:9:373-80.

31. Uzochukwu BS, et al. Determinants of non-adherence to subsidized antiretroviral treatment in Southeast Nigeria. Health Policy Plan. 2009:24(3):189-96.

32. Thao VP, et al. Second-line HIV therapy outcomes and determinants of mortality at the largest HIV referral Center in Southern Vietnam. Medicine (Baltimore). 2015;94(43):e1715.

33. Pujades-Rodriguez $\mathrm{M}$, et al. Treatment failure and mortality factors in patients receiving second-line HIV therapy in resource-limited countries. JAMA. 2010;304(3):303-12

Ready to submit your research? Choose BMC and benefit from:

- fast, convenient online submission

- thorough peer review by experienced researchers in your field

- rapid publication on acceptance

- support for research data, including large and complex data types

- gold Open Access which fosters wider collaboration and increased citations

- maximum visibility for your research: over $100 \mathrm{M}$ website views per year

At BMC, research is always in progress.

Learn more biomedcentral.com/submission 